\title{
EL RENDIMIENTO ESCOLAR Y EL PROBLEMA DE IGUALDAD DE OPORTUNIDADES
}

\author{
Guillermo Mojica D.
}

\section{El problema de la igualdad de oportunidades y la igualdad de resultados}

Aunque tradicionalmente en nuestro medio las gentes afirman creer en el principio de la igualdad, cuando se les exige que expliquen el significado del término, sus respuestas son por lo general vagas y contradictorias. La realidad es que por encima de los principios constitucionales la mayoría acepta como un hecho una situación de desigualdad en casi todas las esferas de la vida diaria, pero cree que debe existir igualdad de oportunidades. Esto es, que las reglas de juego que determinan quiénes son exitosos y quiénes no, deben ser equitativas. Aunque obviamente surgen discrepancias sobre lo que es y no es equitativo, se mantiene vigente el principio universal de la competencia justa, o de lo que los norteamericanos denominan el "fair play". Este principio está íntimamente ligado en nuestro tipo de sociedad con la responsabilidad constitucional que tiene el estado de ofrecer igualdad de acceso y de posibilidades a toda la población, al sistema educativo. En el contexto de los países occidentales que surgen a partir de la revolución francesa, la educación se ha considerado como el instrumento básico para el logro de la igualdad de la igualdad de oportunidades. Históricamente se ha mantenido la creencia de que la educación "es el gran igualizador de la condición del hombre", que hace posible la movilidad de los pobres y desvalidos hacia los beneficios económicos y sociales del progreso y la democracia.

La versión más común de este tipo de argumentación consiste en asegurarle a todas las personas que entran al mercado de trabajo, destrezas comparables. El sistema educativo supuestamente logra darles a todos por igual, destrezas cognoscitivas, lo que permite que todos tengan las mismas oportunidades para competir como adultos. Este tipo de razonamiento descansa en los siguientes supuestos básicos:

a) La eliminación de la pobreza es por lo general un problema de ayudar a la niñez pobre a salir de pobre. Una vez que estas familias escapan de la pobreza, no vuelven a ser pobres. Los hijos de las clases medias por lo general pocas veces son pobres.

b) La razón fundamental por la cual la niñez pobre no puede salir de la pobreza es porque carece de educación y por ende de las destrezas cognoscitivas básicas para competir en el mercado de trabajo, viéndose obligados a permanecer en oficios mal remunerados.

e) El mejor mecanismo para romper con el círculo de la pobreza es a través de reformas educativas. Como la niñez de las familias pobres no puede adquirir estas destrezas, debe ofrecérselas la escuela y el colegio.

d) La educación logra este objetivo ofreciendo igualdad de acceso a toda la población y un rendimiento escolar comparable en todos sus estudiantes. 
Esta creencia en el caso de América Latina tiene profundas raíces históricas y encuentra sus más notables voceros en Sarmiento (Argentina), Bello (Chile), López de Mesa (Colombia) para quienes la educación tenía una misión salvadora y era requisito indispensable para el desarrollo de la nacionalidad y la vida democrática. Para alcanzar estos propósitos de igualdad de oportunidades era necesario tener un sistema de educación universal, obligatorio y gratuito ${ }^{92}$. Buena parte de esta tradición presupone que el problema de la pobreza es un problema hereditario; que la principal razón por la cual unas personas terminan mas ricas que otras es porque desarrollaron mayores destrezas cognoscitivas y que la escuela puede reducir sustancialmente las desigualdades cognoscitivas de la población.

Desde el punto de vista de la teoría educacional, la controversia se centra en preguntarse, si el hombre es el resultado de sus primeras experiencias o de las experiencias escolares. Quienes pregonan por un papel central del sistema educativo en el logro de una igualdad en el desarrollo de las destrezas cognoscitivas, colocan toda la importancia en la etapa escolar, es decir en el período entre los 7 y los 18 años de edad. Al parecer existe hoy un consenso respecto a la mayor importancia que se le otorga en el desarrollo del potencial humano, a las primeras experiencias, en contraposición con una ya larga tradición que ha favorecido el enfoque de la etapa escolar. Este es el primer punto que debe ser esclarecido en el propósito de diseñar un marco de referencia intelectual que sirva para ubicar el mérito de un enfoque investigativo.

El segundo aspecto que se requiere clarificar es la incidencia del medio ambiente en el rendimiento escolar. Desde fines del siglo pasado, ante el fracaso de las reformas educativas se establecieron sistemas de evaluación y seguimiento que reforzaron el prejuicio de clase, atribuyéndole el fracaso del estudiante a su origen o clase social. En la última década y como consecuencia de reformas educativas que surgieron de mandatos contra la pobreza, ha sido tema central de controversia intelectual y el fracaso de la educación en la búsqueda de la igualdad de oportunidades. Concretamente en países desarrollados como en los Estados Unidos estas políticas han buscado eliminar la pobreza, ofreciéndole a los grupos más pobres oportunidades a través de la educación para mejorar su condición competitiva frente a otros grupos en el mercado de trabajo. Nuevamente se ha puesto en boga el papel de la educación, esta vez concebida dentro del enfoque de la factoría. Aunque es verdad que las escuelas tienen "insumos" y "productos" y que uno de sus propósitos es el de producir la "materia prima humana", este enfoque supone que el rendimiento escolar depende fundamentalmente de factores ambientales, económicos y genéticos y que es posible aislar sus efectos nocivos para optimizar la asignación de los recursos escolares.

La controversia contemporánea se ha centrado nuevamente sobre el mismo tema y una vez mas se ha puesto en tela de juicio el modelo de la factoría escolar como herramienta en el logro de la igualdad de oportunidades. Luego de una década de investigación sistemática sobre el tema del rendimiento escolar, existe un consenso unánime que le otorga a las características del alumno que ingresa a la escuela a un peso definitivo en el resultado que se obtiene al final del proceso escolar. Esta afirmación tiene implicaciones básicas para los educadores: La primera, que lo que importa desde el punto de vista del desarrollo humano es lo que ocurre al niño antes de los siete años que es la edad para ingresar a la escuela. Segundo, que durante el proceso escolar, la incidencia de sus antecedentes familiares, de su grupo de referencia, de su nivel de inteligencia, del tamaño

\footnotetext{
${ }^{92}$ Darcy Ribeiro. Las Américas y la Civilización, cuadernos latinoamericanos. Centro Editor de América Latina, 1969.
} 
del aula, del presupuesto de la escuela, del currículum o calidades del profesor, tienen un efecto secundario y por lo general irrelevante en el producto escolar. El rendimiento escolar está determinado por lo que le ocurrió al niño antes de ingresar a la escuela. En este sentido, el problema de la desigualdad de oportunidades trasciende el medio educativo y debe considerarse dentro de los alcances de una política social.

Desde la perspectiva de la política social, el esfuerzo intelectual de la última década ha dejado sin piso la creencia de que la igualdad en la educación y en el desarrollo de destrezas cognoscitivas juega un papel significativo en la distribución del ingreso. Es importante recalcar que este modelo no tuvo como punto de partida la idea de maximizar la satisfacción del consumidor, sino que partió del supuesto de que una igualdad en las oportunidades educativas era necesaria para el logro de una igualdad en el ingreso y el prestigio social.

La contribución intelectual es clara en el sentido de que las relaciones entre educación y destrezas cognoscitivas de una parte y nivel de ingreso y prestigio ocupacional de la otra, son poco significativa, más aún, que existen pocas relaciones entre las diferentes variables que se supone determinan el ingreso, esto es, que la distribución de credenciales educativas, el prestigio ocupacional y el nivel de ingresos, están desigualmente distribuidos y poco relacionados entre sí. Lo anterior significa que ninguna estrategia individual puede atacar simultáneamente las distintas dimensiones de la desigualdad. Tendrían que diseñarse una variedad de acciones específicas para tratar cada una de las desigualdades específicas. Más aún, lo anterior indica que las premisas que han sustentado la acción política en la interpretación del problema son equivocadas. El problema desde una perspectiva de reforma estructural no consiste en disminuir la capacidad de unos individuos de obtener beneficios o ganancias comparativas a costa de otras persona, sino en modificar las reglas del juego para reducir los beneficios del éxito competitivo de unas minorías y el costo del fracaso de unas mayorías. Antes de entrar a este punto y de plantear las implicaciones que tendría este enfoque para un programa de investigaciones, es necesario complementar el análisis crítico del modelo educacional desde una perspectiva histórica.

\section{La educación y la desigualdad de oportunidades: breve reseña histórica}

El principio de la escuela pública adquirió aceptación general en el mundo occidental a mediados del siglo pasado, con la creencia de que un sistema de educación pública cumpliría objetivos más allá de la simple instrucción. Los reformistas de la época insistían en el principio de que la escuela pública podría convertirse en un agente de reforma y de disciplina social. Visto de otra manera, mantenían la esperanza de que la educación promovería el orden social desestimulando ambiciones que no correspondieran con la realidad y perspectivas de los estudiantes. Ambos aspectos llegaban a la misma conclusión: el mejor interés de la sociedad se encontraba en un sistema de educación pública que aislara al estudiante de otras influencias y lo condujera a lo largo de un régimen regular, dirigido por una burocracia profesional centralizada ${ }^{93}$.

La idea de los derechos de la infancia es un concepto relativamente reciente en la historia. En la época medieval no existía la adolescencia ${ }^{94}$. El concepto de la adolescencia, como una época específica, como un fenómeno tanto sicológico y social como biológico, simplemente es de años recientes. Anteriormente el párvulo pasaba directamente de la infancia a la vida adulta. El cambio comenzó en el Siglo XVIII cuando

\footnotetext{
${ }^{93}$ Philippe Aires. Centuries of Childhood. Vintage Books, New York. 1962.

${ }^{94}$ Philippe Aires, obra citada.

Digitalizado por RED ACADEMICA
} 
se introdujo el concepto rousseanniano del "infante inocente" que tenía que ser confinado o aislado de la corrupción del mundo circundante y concretamente del ambiente de vicio y depravación de las masas ${ }^{95}$. El resultado fue la invención de la escuela pública como una institución especializada para la instrucción de la población, cuidadosamente aislada del resto de la sociedad.

El movimiento reformista que consideraba el aislamiento como un medio de curación, culminó con el aislamiento de la niñez como un grupo social que requería de su propio asilo. La escuela pública aparece como un refugio dominado por normas de control burocrático, de desconfianza y de castigo ${ }^{96}$.

El principio de la educación pública permanece hasta buena parte del Siglo XIX corno una intención más que una realidad y su implantación requirió de legislación compulsoria y de prohibiciones sobre el trabajo de menores para persuadir a las masas de aceptar este principio.

Históricamente se ha exagerado el concepto publico o popular de la educación pública, ya que en su origen es una institución esencialmente orientada hacia los estratos medio y altos. Históricamente en donde ha existido considerable homogeneidad de clase social, de religión o de antecedentes étnicos, las escuelas han sido eficaces en términos de resultados ${ }^{97}$.

La educación pública nunca ha sido eficaz cuando prevalece la heterogeneidad de su clientela, y a los sectores populares siempre les ha servido mal. Entre otras razones porque la educación no fue un medio importante de movilidad en el desarrollo de los países industrializados. Por el contrario, la movilidad, en ocasiones, se logró sacrificando la educación de la generación joven. Los salarios eran tan bajos y el desempleo tan frecuente que los migrantes, en su mayoría trabajadores no calificados, dependían tanto de sus ingresos, como de los de sus hijos y de su esposa ${ }^{98}$. Para cualquier observador del medio colombiano, esta situación no es muy distinta de la que se observa actualmente en nuestros centros urbanos.

De otra parte, la experiencia educativa y en el contexto del nuevo continente, desde el punto de vista del currículum y de los métodos de enseñanza, partió de la implantación de las escuelas europeas que fueron diseñadas para instruir a las clases altas ${ }^{99}$; pocos han sido de nuestro medio los intentos de conocer las necesidades especiales de la población de los estratos populares. La experiencia colombiana a través del tiempo es un buen ejemplo de esta tradición, de ahí que no debe sorprender a nadie el prejuicio social que caracteriza el sistema educativo colombiano. En teoría, la escuela pública es para todos; en la práctica, el sistema se rige por un conjunto burocrático de normas rígidas, de costumbres y tradiciones que tienen como consecuencia mantener aisladas a la población para cuyo beneficio fueron originalmente establecidas ${ }^{100}$.

Hacia fines del Siglo XIX, la reforma escolar concebida dentro de la anterior perspectiva

\footnotetext{
${ }^{95}$ Christopher Lasch. The World of Nations, Vintage Books, New York, pág. 13.

${ }^{96}$ Colin Greer. The Great School Legend: A Revisionist Interpretation of American Public Education Basic Books, New York, pág. 207.

${ }^{97}$ Charles E. Silverman. Crisis in the Classroom, Random House, N.Y. 1970. pag. 54.

${ }^{98}$ Charles E. Silverman, obra citada, pag. 55.

${ }^{99}$ Lawrence A. Cremin The Genius of American Education, Vintage Books, N Y. 1966.

${ }^{100}$ Michael B. Katz. The Irony of Early Educational Reform, Harvard University Press. Cambridge, 1968.
} 
fue centro de una enorme controversia pública. Las escuelas eran insuficientes y costosas; el proceso de enseñanza repetitivo y memorizante; los desertores y repitentes se multiplicaban. Estas críticas nunca tuvieron como propósito atacar los principios de la educación gratuita y universal, sino mas bien hacer las escuelas más eficientes.

Como respuesta al fracaso de los sistemas educativos en la instrucción de la niñez de los sectores pobres, se establecieron sistemas de evaluación y seguimiento que tuvieron como efecto orientar el fracaso académico de esta población escolar, hacia programas de manualidades y orientación vocacional. Para quienes hayan seguido las políticas educativas en Colombia durante la última década, la similitud de enfoques no obedece a simples coincidencias.

\section{El aporte científico al problema del rendimiento}

En los Estados Unidos, a partir de mediados de la década del 60 y como consecuencia de decisiones que se tomaron sobre la política pública, se formularon e implementaron políticas para eliminar las desigualdades de oportunidades de ciertos grupos étnicos. Igualmente tuvieron como propósito atacar los problemas de pobreza y privación de ciertos grupos sociales y de zonas específicas del país. La atención de estas políticas estuvo orientada a ayudar a aquellas personas en ocupaciones no calificadas y de baja remuneración hacia mejores ocupaciones. La estrategia de estas políticas se basaba en un conjunto de supuestos que, como se mencionó anteriormente, descansaban en el logro de igualdades educativas. Los programas educativos se orientaron a hacer obligatoria la asistencia de la niñez de familias pobres a las escuelas da la población de sectores medios, a programas de educación compensatoria dirigidos a desertores y repitentes, y a una mayor participación de los padres en la administración escolar.

La estrategia tuvo como soporte una investigación realizada en 1966 por el Profesor James Coleman sobre la "Igualdad de Oportunidades Educativas", que constituye el primer intento de medir el rendimiento escolar.

Sin duda alguna es una investigación clásica; la de mayor cobertura y rigor intelectual que hasta entonces se hubiera realizado en el mundo y que es centro de controversia intelectual y punto de partida necesario para cualquier esfuerzo investigativo sobre el tema. A partir de esta fecha, se han desarrollado investigaciones posteriores de seguimiento y revisión de los esquemas teóricos originales, que conforman un cuerpo de conocimientos teóricos y de resultados empíricos, que hoy permiten disponer de un marco de referencia que ninguna investigación seria puede desconocer.

Siendo el propósito de este ensayo ofrecer un marco de referencia intelectual que le aporte elementos de juicio a la Fundación en la definición de una política investigativa en el campo de la educación, se presenta a continuación una síntesis del alcance y la contribución que estas investigaciones, que denominamos clásicas, aportan al conocimiento científico y a las acciones concretas de la política pública.

\section{A. El informe Coleman}

Exámenes para medir el conocimiento en escritura, lectura, matemáticas y solución de problemas, fueron realizados con base en una muestra de 645.000 alumnos de los cursos $1,3,6$ y 10 de cerca de 4.000 escuelas y colegios, que cubrían todos los estados y el distrito de Columbia. Los exámenes no medían inteligencia, actitudes o carácter; se orientaron a la medición de destrezas y conocimientos, considerando que "estos factores eran más significativos en la obtención de una buena ocupación y en la posibilidad de 
promoción ocupacional en un contexto cada vez más técnico". Por consiguiente, se supuso que los resultados al final del proceso escolar eran un buen indicio del rango de oportunidades que tenía el alumno al final de su educación.

Los resultados centrales de la investigación fueron los siguientes:

1. Los resultados para el estudiante promedio de los grupos étnicos de indios americanos, mexicano-americanos, negros y puertorriqueños, fueron sustancialmente inferiores al obtenido para el estudiante promedio blanco.

\section{Las disparidades se amplían a lo largo del proceso escolar.}

3. Estas no pudieron ser explicadas por las diferencias en la calidad de la educación ofrecida por los colegios que asistían a los grupos minoritarios.

4. Se partió del supuesto contrario de que las diferencias se debían a la mala calidad de la educación recibida en escuelas y colegios no con menores recursos y presupuestos, que se reflejaban en variables como la antigüedad de las edificaciones, el número de textos, facilidades de bibliotecas, tamaño promedio del grupo y nivel educativo del maestro.

Se supuso entonces que estas desigualdades en la calidad educativa explicarían las diferencias del rendimiento escolar, dentro del modelo de que la diferencia de insumos explica la diferencia del producto. Sobre esta base se había diseñado la política pública vigente.

5. El aspecto critico de la investigación es el de haber demostrado que las diferencias de la calidad de la educación estaban muy poco relacionadas con el rendimiento escolar. La relación de causa y efecto simplemente no se materializó

6. Finalmente encontraron que las diferencias en el rendimiento escolar parecen estar más relacionadas con los antecedentes familiares del alumno que con diferencias en la calidad de la educación. Más aún, se encontró que el colegio poco contribuye a modificar la influencia que el medio ambiente familiar tiene en el rendimiento escolar.

\section{B. El informe del seminario sobre igualdad de oportunidades educativas, Universidad de Harvard, 1967}

Las conclusiones de la investigación de Coleman pusieron en tela de juicio viejas creencias sobre la función de la escuela, que tiene raíces muy arraigadas, no sólo entre educadores, sino en general a nivel de la sociedad. Estos antecedentes y el relativo fracaso de las políticas contra la pobreza, trazadas en base al papel crítico de la educación en el logro de una mayor igualdad de oportunidades, convirtió el estudio Coleman en el documento quizás más polémico y criticado desde el Dilema Americano de Myrdal. Las críticas centrales se refirieron a los defectos de la muestra, ya que no sólo hubo colegios que rehusaron participar sino otros que colaboraron a medias, dejando de entregar parte de la información. Resultados completos sólo fueron recibidos del $60 \%$ de los colegios originalmente incluidos dentro de la muestra.

Igualmente los autores han sido criticados por el uso de técnicas estadísticas de dudosa comprobación y por errores en el manejo de los datos. 
La Universidad de Harvard inició en 1967 un seminario sobre lgualdad de Oportunidades Educativas que tuvo como propósito volver a examinar los resultados del informe Coleman. El resultado de su trabajo fue el de corroborar las conclusiones iniciales y anotar "que el efecto neto de los errores estadísticos del informe original fue el de subestimar la importancia de los antecedentes familiares y de sobre valorar la importancia de la escuela en la determinación del rendimiento académico ${ }^{101}$.

\section{El informe Jenks}

En 1968 y como consecuencia de los dos proyectos anteriores, se creó el Centro para la Investigación de la Política Educativa en la Universidad de Harvard, el cual inició un esfuerzo más amplio en el estudio de las relaciones entre el rendimiento escolar, las desigualdades económicas y las estrategias contra la pobreza. Se trataba del primer intento sistemático de relacionar las políticas educativas con las políticas sociales.

La investigación tuvo como propósito demostrar que las estrategias tradicionales para equiparar el poder adquisitivo entre individuos no operan. Los aspectos centrales del análisis son:

a) Un examen del alcance de las desigualdades en la educación, tomando variables tales como: Los presupuestos escolares, su composición social y el acceso de los estudiantes al currículum de su escogencia.

b) Un análisis de la influencia de los factores hereditarios y ambientales incluyendo las escuelas en los resultados de los exámenes de rendimiento escolar.

c) Un examen de los factores que determinan o tienen influencia en la distribución de credenciales educativas.

d) Finalmente investigaron los factores que determinan el prestigio ocupacional y el ingreso, entre los cuales tomaron en cuenta los antecedentes familiares, las destrezas cognoscitivas, las credenciales educativas y la calidad de los establecimientos escolares.

Las conclusiones centrales del estudio fueron las siguientes: Las oportunidades educativas, las destrezas cognoscitivas, las credenciales educativas, el prestigio ocupacional, el ingreso y la satisfacción en el cargo están todas desigualmente distribuidas. La asociación entre estas variables "es usualmente muy débil lo cual significa que intentar una mayor igualdad en una de estas variables poca incidencia tiene en las demás" ${ }^{\prime 102}$.

Sobre el aporte de la educación y específicamente sobre la relación entre el rendimiento escolar y la igualdad de oportunidades los resultados corroboraron las conclusiones de los proyectos anteriores:

1. El acceso a las oportunidades educativas era muy desigual. La utilización de los recursos escolares entre establecimientos era todavía más desigual, sobre todo a partir de la edad de 16 años.

\footnotetext{
${ }^{101}$ Equal Educational Opportunity. Harvard Educational Review, Harvard University, Cambridge.1969.

${ }^{102}$ Christopher Jenks. Inequality, Basic Books Inc. N.Y. 1972 pp. 253. 
2. Las desigualdades genéticas y ambientales juegan un papel significativo en la reproducción de desigualdades cognoscitivas. Además, aquellos que en sus primeros años de vida tuvieron ventajas genéticas, lograron igualmente ventajas ambientales agudizando las desigualdades.

3. No encontraron ninguna evidencia sobre el supuesto de que la diferencia de calidad de los establecimientos educativos contribuyeran a las desigualdades cognoscitivas.

4. Los antecedentes familiares tienen mucha más influencia que los genéticos en la perseverancia escolar, es decir, en el número de años de estudio de la población. La influencia de la familia depende en parte de su nivel socioeconómico y en parte, en características culturales y sicológicas que son independientes del nivel económico.

5. Las diferencias cualitativas de las escuelas juegan un papel muy secundario en la determinación de cuántos años de estudio eventualmente logra la población.

6. El prestigio ocupacional está muy ligado al número de años de estudio y por consiguiente a las credenciales educativas. Los antecedentes familiares y las destrezas cognoscitivas, tienen influencia sobre el prestigio ocupacional, porque inciden en la cantidad de educación que obtienen las personas y no por su influencia en el prestigio personal de los individuos que hayan culminado su educación. La calidad de la educación primaria y secundaria tiene poca influencia en la determinación del prestigio ocupacional.

7. La variación de los ingresos de las personas resultó muy difícil de explicar. Al parecer existe tanta variación en el ingreso entre personas provenientes de familias similares, con credenciales y rendimiento escolar similar, como la que existe entre el total de la población.

Antes de entrar a plantear las implicaciones de los anteriores resultados, es importante complementar la breve reseña con otros proyectos, algunos realizados en otros países incluyendo a Colombia, ya que nos permite tener un universo mas amplio para demostrar la validez universal de los resultados y de sus implicaciones para la política pública y la tarea investigativa.

\section{El informe Plowden}

En Inglaterra en 1968 se inició un estudio comparable al informe Coleman, que evitó en buena parte las críticas y errores metodológicos que a éste se le atribuyen, habiendo llegado a conclusiones similares ${ }^{103}$.

\section{E. El informe Burkhead}

Con base en la técnica de insumo-producto, el profesor Burkhead construyó las relaciones entre la asignación de recursos y otros insumos escolares y el producto medido a través de exámenes de conocimientos de una muestra de colegios de bachillerato de cinco grandes ciudades de los Estados Unidos. El resultado central de este proyecto fue el de que "las variaciones en el rendimiento escolar, estaban casi totalmente determinadas o condicionadas por el nivel socio-económico del vecindario"104.

El nivel de ingreso del barrio, las condiciones de la vivienda, la ocupación de los

\footnotetext{
${ }^{103}$ Central advisory Council For Educations. Children and Their Primary Schools, London: her Majesty's Stationary Office 1967.

${ }^{104}$ Jesse Burkhead with Thomas Fox and John W. Holland, Imput and output in Large-City High Schools, Syracuse University Press, 1967.
} 
padres, el origen étnico, son las variables importantes que determinan el rendimiento escolar.

\section{F) El informe Stephems}

Se trata de una investigación para el estudio sistemático del proceso de enseñanza, que recoge información de varios estudios sobre el mismo tema y concluye afirmando que el rendimiento escolar no se modifica por el tamaño del aula, el tamaño del colegio, la organización administrativa de la institución, el promedio de tiempo dedicado a la instrucción, la habilidad del maestro, la personalidad del profesor y los métodos de enseñanza ${ }^{105}$.

\section{G. La contribución de la Misión Alemana en Colombia}

En 1965 llegó al país una misión de expertos alemanes, para colaborar con el Ministerio de Educación en la capacitación de maestros y mejoramiento de la enseñanza. La primera tarea fue la conformación de un grupo mixto para adelantar una investigación a nivel nacional sobre la calidad de la enseñanza. Se tomó una muestra de 70.000 estudiantes de escuelas primarias, distribuidos en los cinco grados y por zonas geográficas, a quienes se les hicieron los exámenes de conocimientos en matemáticas y lenguaje, tal como lo establecían las respectivas normas oficiales. Se trataba de conocer hasta qué punto los niños aprendían las enseñanzas que, según el decreto oficial, deberían impartir los maestros.

Se partió de una metodología que permitía identificar por separado los dos aspectos o dimensiones del proceso cognoscitivo del aprendizaje. La capacidad del estudiante, de abstraer y conceptualizar el problema y la habilidad para utilizar las técnicas e instrumentos en la solución del mismo. Lo primero permitía medir la inteligencia del niño; lo segundo, indicaba la contribución instrumental de la escuela.

Los resultados de la investigación mostraron una familia de curvas que medía la inteligencia y las destrezas, cuyos vértices reflejaban niveles de inteligencia satisfactorios y niveles de destrezas muy pobres. Dicho en otros términos, el niño colombiano tiene un nivel de inteligencia aceptable, pero su rendimiento escolar era muy deficiente en comparación con la experiencia que se obtuvo con la misma metodología en Alemania. Adicionalmente se encontró que durante el transcurso escolar de la primaria, ambas curvas decrecían.

Se hicieron estas mismas pruebas con base en una muestra de 2.500 maestros y se encontró que para el caso de matemáticas, el resultado fue en promedio de 1 en una ponderación de 1 a 5, y de 2 en el caso del área de lenguaje.

\section{Implicaciones de las investigaciones para la política pública y para el desarrollo de la investigación educativa en Colombia}

Los resultados del esfuerzo científico sobre el tema del rendimiento escolar y de sus relaciones con las políticas del Estado, para lograr una mayor igualdad de oportunidades y eliminar la pobreza, tienen sustanciales implicaciones para aquellas personas que como investigadores quieran contribuir en nuestro medio a una mayor comprensión del problema. Como en toda labor científica, el problema no es de técnicas o simple

\footnotetext{
${ }^{105}$ J.M. Stephens. The Procers of Schooling, Holt, Rinehart — Winston, New York 1967.
} 
manipulación mecánica de datos.

El acerbo científico de que se dispone afecta la labor tanto de los educadores como de los investigadores sociales.

\section{A. Implicaciones para los educadores}

Empezaremos por hacer referencia de las implicaciones que los resultados Llenen para los educadores.

Ninguna de las investigaciones reseñadas sugiere que de reformas educativas puedan derivarse cambios sustanciales o significativos en la estructura social fuera de la escuela. Más aún, la evidencia empírica sugiere que la igualdad de oportunidades educativas (aparte de que es utópico en nuestro medio alcanzarla) aportaría muy poco al logro de una igualdad de oportunidades de los adultos.

La experiencia de los últimos 25 años sugiere que en los países desarrollados, reducciones sustanciales en las diferencias de credenciales educativas, disminuyen en muy poco las desigualdades económicas entre los adultos.

La utilidad de programas de educación compensatoria tiene por lo general un beneficio marginal para los que la reciben. Ni el nivel total de los recursos educativos, ni los programas específicos claramente definidos a nivel escolar, tienen influencia en el rendimiento escolar para la población de adolescentes que llegó a la escuela con desventajas por sus antecedentes familiares.

Hay por lo menos tres razones centrales por las cuales las reformas educacionales no inciden un una mayor igualdad para la vida adulta. La primera porque la niñez recibe mayores influencias y está más condicionada por lo que le ocurrió en su ambiente familiar antes de llegar a la escuela. Inclusive, porque en nuestro medio urbano, reciben una mayor influencia de lo que ocurre en las calles y la TV. que en el propio colegio. Segundo, porque la naturaleza burocrática del aparato educativo, hace muy difícil que las reformas incidan en aquellos aspectos de la escuela que influyen directamente en la vida diaria del estudiante. Se trata del problema de la transformación de innovaciones en ritualismo burocrático. La asignación a nivel nacional de los recursos, la reubicación de maestros y estudiantes, las modificaciones del currículum casi nunca modifica las costumbres y normas de un sistema concebido para "educar para ser dóciles"106.

Tercero, porque aún en el caso de que el colegio ejerza una influencia significativa en la niñez, ésta es difícil de que perdure en la vida adulta si de otra parte no han ocurrido cambios significativos en el sistema social. Se requieren cambios radicales en los resultados del rendimiento escolar en primera, para modificar significativamente el nivel de ingresos de un adulto.

Los argumentos anteriores sugieren que el modelo de la factoría escolar, concebido como instrumento de una política de redistribución del ingreso, debe ser abandonado. Es cierto que las escuelas tienen "insumos" y "productos" y que uno de sus propósitos nominales, es tomar "la materia prima humana" para convertirla en algo de mayor valor.

El resultado del esfuerzo científico muestra sin embargo que el producto de las escuelas depende fundamentalmente de un solo insumo, esto es, de las características del niño cuando por primera vez llega al sistema escolar. Todo lo demás, el presupuesto

\footnotetext{
${ }^{106}$ Ver Charles E. Silverman, Crisis in the Classroom, Random House, New York. 1970. Capítulo 4, pp. 113 a 157 
de la escuela, sus políticas, su grupo de referencia y sus maestros, son secundarios o simplemente irrelevantes ${ }^{107}$.

Las diferencias de recursos cognoscitivos medidos a través del nivel de inteligencia y del rendimiento escolar, muestran claramente que el $50 \%$ del desarrollo de la inteligencia ocurre antes de los 8 años. Entre los 8 y los 17 años, cuando la persona está en edad escolar, el desarrollo de la inteligencia es sólo de un $20 \%{ }^{108}$ En síntesis, así como la inteligencia se desarrolla tanto en los primeros 4 años de la vida como en los 13 siguientes, se incrementa muy poco luego de los 18 anos.

De igual manera, una tercera parte de la capacidad potencial de rendimiento que el niño logre en la escuela, ya la ha desarrollado durante los primeros cinco años de vida, probablemente porque la mayor parte de esta capacidad potencial depende del desarrollo del vocabulario y de la comprensión del lenguaje ${ }^{109}$.

Si aceptamos que el "mayor valor" que aporta la escuela a la materia prima humana, depende fundamentalmente de las características que trae la población escolar tenemos que reconocer que las posibilidades de una mejor educación, dependen igualmente de lograr una igualdad de oportunidades para la infancia antes de su ingreso a la escuela.

Es necesario restaurar el valor intelectual de la educación y abandonar la búsqueda de una mayor igualdad de oportunidades con el modelo escolar de la factoría. La escuela debe dejarse libre de ataduras reivindicativas o redistributivas para que responda a las necesidades del intelecto. Esto es, que la diversidad sea su centro de interés.

\section{B. Implicaciones para los investigadores sociales}

Para quienes tienen como preocupación central la desigual distribución del ingreso, el resultado de las investigaciones reseñadas tiene implicaciones más negativas que positivas. El enfoque indirecto para atacar la pobreza y las desigualdades, poco porvenir tiene si nos atenemos tanto al resultado investigativo como al fracaso de las políticas implantadas a través de la estrategia educativa en los últimos 25 años en diversos países, entre los cuales tenemos que incluir a Colombia. Se trata de eliminar la pobreza absoluta y las desigualdades, esto requiere una acción directa del Estado. Diversos caminos se han explorado, algunos se han implantado y seguramente nuevos aparecerán en el futuro.

\footnotetext{
${ }^{107}$ Christopher, Jenks, Inequality, obra citada, pag. 256.

${ }_{108}$ Benjamin Bloom. Stability and Change in Human Characteristics, John Wileyand Sons. New York. pag. 237.

${ }^{109}$ Ibid. pp. 247.
} 